Cuestiones de filosofía

ISSN: 0123-5095

Vol. 1 - No. 18

Enero - junio, año 2016

pp. $89-108$

\title{
GUBERNAMENTALIDAD: De la tradición biopolítica a la tradición gubernamental ${ }^{1}$
}

\section{GOVERNMENTALITY: From biopolitics tradition to governmental tradition}

\author{
Hernán Dario Ocampo-Giraldo ${ }^{2}$ \\ Universidad del Rosario, Colombia
}

Fecha de recepción: 6 de enero de 2016

Concepto de evaluación: 28 de marzo de 2016

Fecha de aprobación: 15 de julio de 2016

\section{Resumen}

El propósito de este comentario sobre la tradición biopolítica es ayudar a aclarar, a los lectores nuevos en estas cuestiones, el panorama general y la ruta que se ha trazado en torno a los análisis de esta tecnología que describió Foucault. En este artículo se sostiene que hay, al menos, cuatro momentos claramente identificables dentro de la tradición biopolítica, y tres dentro de la tradición gubernamental; ambas tradiciones están asociadas al contexto social que demanda nuevas teorías para analizar el presente, así como a la divulgación de esas teorías. También se traza aquí una grilla de inteligibilidad entre la biopolítica y la gubernamentalidad.

Palabras clave: Anatomopolítica. Biopoder. Biopolítica. Estudios gubernamentales. Gubernamentalidad. Tanatopolítica.

\footnotetext{
${ }^{1}$ Investigación realizada en el Semillero de Investigación en Biopolítica y Gubernamentalidad, durante el 2015. Este semillero interinstitucional pertenece a las universidades de La Salle y El Rosario, Bogotá.

${ }^{2}$ Hernán Darío Ocampo Giraldo. Magíster en Filosofía y Licenciado en Filosofía y Letras. Profesor de las Universidades El Rosario y La Salle. Director del Semillero de Investigación en Biopolítica y Gubernamentalidad. ocampo.hernan@urosario.edu.co.
} 


\begin{abstract}
The purpose of this comment, about the biopolitics tradition, is to help new readers on these issues, to clarify the overall picture and the path that has been set about the different types of this technology analysis, described by Foucault. This article argues that there are at least four clearly recognizable moments in the tradition of biopolitics and three within the governmental tradition. Both traditions are associated with the social context that demands new theories to analyze the present, and the dissemination of these theories. Also it traces a grid of intelligibility between biopolitics and governmental conditions.
\end{abstract}

Key words: Anatomopolitic. Biopolitic. Biopower. Governmentality. Governmentality Studies. Tanatopolitic.

\title{
LA TRADICIÓN BIOPOLÍTICA
}

Tal vez la filosofía pueda cumplir aún un papel por el lado del contrapoder, con la condición de que ese papel ya no consista en hacer valer, frente al poder, la ley misma de la filosofía, de que esta deje de pensarse como profecía, deje de pensarse como pedagogía o legislación y se asigne la tarea de analizar, dilucidar, hacer visibles y por lo tanto intensificar las luchas que se libran en torno del poder, las estrategias de los adversarios en el seno de las relaciones de poder, las tácticas utilizadas, los focos de resistencia; con la condición, en suma, de que la filosofía deje de plantear la cuestión del poder en términos de bien o mal, y lo haga en términos de existencia (Foucault, 1978) ${ }^{3}$

La tradición biopolítica presenta varios momentos (ver cuadro 1); el primero es el análisis realizado por Foucault durante la década de los setenta. Los lectores podrán encontrar los siguientes conceptos en los textos anotados junto a ellos: tanatopolítica: El poder psiquiátrico. Curso en el Collège de France (19731974), [clases del 7-14-21-28 de noviembre]; Los Anormales. Curso en el Collège de France (1974-1975) [clases del 8, 15, 22 de enero]; Vigilar y

\footnotetext{
${ }^{3}$ Con esto se refiere al postulado de Seguridad, territorio, población (STP) de NO HACER POLÍTICA, a que el campo de lucha de la filosofía misma está en mostrar la relación entre los efectos de saber y las tácticas de la verdad que se producen en un momento, periodo y campo determinado, en un campo de fuerzas reales; en este sentido el filósofo hace su lucha, este es su campo de batalla, y no es, entonces, el campo de la política, si por ella entendemos toda la constitución del aparato gubernamental y legislativo.
} 
castigar [Suplicio, Castigo]. Anatomopolítica: Los Anormales. Curso en el Collège de France (1974-1975) [clases del 29 de enero y del 5 y 12 de febrero]; Vigilar y castigar [Disciplina, Prisión]. Biopolítica y Biopoder: Defender la sociedad. Curso en el Collège de France (1975-1976) [clase del 17 de marzo]; Historia de la sexualidad 1: La voluntad de saber [Capítulo V: Derecho de muerte y poder sobre la vida]; Seguridad, territorio, población. Curso en el Collège de France (1977-1978) [Clase del 11, 18, 25 de enero]; Nacimiento de la biopolítica. Curso en el Collège de France (1978-1979). [Como proyecto]. Este primer momento es cercano a las primeras declaraciones que hace Foucault sobre la biopolítica en La voluntad de Saber y la difusión oral de los cursos del Collège de France, con unas pocas publicaciones de los resúmenes del curso - en este periodo no habían sido publicados como textos completos-.

Foucault abandonó a finales de 1979 el concepto biopolítica y, en general, su discurso pasó de una lectura bélica ${ }^{4}$ de las relaciones de poder a una interpretación de ellas como juego de acciones sobre acciones (CastroGómez, 2010). En la década de los ochenta, las interpretaciones de la obra de Foucault tienden hacia las cuestiones de la ética y la estética, y no hacia las cuestiones de las tecnologías políticas o de las racionalidades. Finalizando la década de los noventa y empezando el nuevo siglo, las cuestiones políticas vuelven a adquirir relevancia en las interpretaciones de la obra de Foucault; en especial, las reflexiones de Agamben en Homo Sacer I (1998) y Medios sin Fin (1996), las de Negri y Hardt en Imperio (2000) y un artículo de Deleuze: «Postescritum a las sociedades de control» (1996) buscaban volver a pensar los textos de Foucault en clave biopolítica, a través del análisis de una forma de poder que tomó la vida como su objeto primordial.

La publicación de los cursos: Defender la sociedad (2002), Seguridad, territorio, población (2006) y Nacimiento de la Biopolítica (2004) ayudaron a que se difundieran los estudios sobre la biopolítica. En la primera década del

\footnotetext{
${ }^{4}$ Esta actitud bélica la describe Castro-Gómez en el primer capítulo de Historia de la gubernamentalidad, y es su grilla de lectura del cambio temático dado por Foucault a partir de la cuarta clase de Seguridad, territorio y población y en todo El nacimiento de la biopolítica. Quien quiera conocer más sobre este cambio remítase a Defender la Sociedad (clase del 7 de enero de 1976), Michel Foucault y Michel Foucault y sus contemporáneos, de Didier Eribon.

${ }^{5}$ Las ediciones que cito son las originales, en la bibliografía aparecen las ediciones de consulta, algunas de las cuales difieren en los años citados.
} 
2000 y lo que llevamos de esta, los trabajos que utilizan esa noción para describir casos concretos se han incrementado de forma sorprendente. Los nuevos investigadores deberían conocer algunos libros clásicos de esta década: Políticas del acontecimiento, de Maurizio Lazzarato (2006); Bíos. Biopolítica y filosofia (2006) y Comunidad, inmunidad y biopolitica (2009), de Roberto Esposito; Biopolitica y formas de vida, editado por Rubén Sánchez (2007); La fábrica de porcelana, de Negri (2008); Commonwealth: El proyecto de una revolución del común (2011), de Negri y Hardt, e Historia de la gubernamentalidad, de Castro-Gómez (2010).

Incluso, podríamos decir que hay un cuarto momento en la tradición biopolítica; sin embargo, no es posible establecer una cantidad determinada de archivos en este periodo (2010 hasta ahora), ni siquiera representativo, de los trabajos sobre este concepto. A pesar de ello, es posible sostener que todos tienen una generalidad: usan las nociones Tanato, anatomo, biopolítica y biopoder para describir casos concretos, los cuales son de amplio espectro, van desde la didáctica y la pedagogía, la medicina, hasta la política, etc. Al respecto se puede observar el volumen de trabajos presentados en el IV Coloquio Latinoamericano de Biopolítica y el II Coloquio Internacional de Biopolítica y Educación, realizados en Bogotá en septiembre de 2013, así como la página www.biopolitica.org. Cada día se incrementa exponencialmente el número de investigaciones e investigadores.

Cuadro 1. La tradición biopolítica

\begin{tabular}{|c|c|c|c|c|c|}
\hline \multicolumn{6}{|c|}{ LA TRADICIÓN BIOPOLÍTICA } \\
\hline Primer momento: & Foucault, setentas & & $\begin{array}{l}\text { Segundo } \\
\text { momento: } \\
\text { noventas }\end{array}$ & $\begin{array}{l}\text { Tercer momento: primera } \\
\text { década del } 2000\end{array}$ & $\begin{array}{l}\text { Cuarto momento: segunda década del } \\
2000\end{array}$ \\
\hline Tanatopolítica & Anatomopolítica & $\begin{array}{l}\text { Biopolítica y } \\
\text { Biopoder }\end{array}$ & & & \\
\hline $\begin{array}{l}\text { El poder } \\
\text { psiquiátrico } \\
(1973-1974) \\
\text { [Clases del 7- } \\
\text { 14-21-28 de } \\
\text { noviembre]; Los } \\
\text { Anormales } \\
\text { (1974-1975) } \\
\text { [clases del } \\
8,15,22 \text { de } \\
\text { enero]; Vigilar y } \\
\text { castigar } \\
\text { ["Suplicio", } \\
\text { "Castigo"]. }\end{array}$ & $\begin{array}{l}\text { Los Anormales } \\
\text { (1974-1975) } \\
\text { [clases del } 29 \mathrm{de} \\
\text { enero, } 5 \text { y } 12 \mathrm{de} \\
\text { febrero]; Vigilar } \\
\text { y castigar } \\
\text { ["Disciplina", } \\
\text { "Prisión"]. }\end{array}$ & $\begin{array}{l}\text { Defender la } \\
\text { sociedad (1975- } \\
\text { 1976)[clase del } 17 \\
\text { de marzo]; La } \\
\text { Voluntad de saber } \\
\text { ["Derecho de } \\
\text { muerte y poder } \\
\text { sobre la vida"]; } \\
\text { Seguridad, } \\
\text { territorio, } \\
\text { población (1977- } \\
\text { 1978) [clase del } \\
\text { 11,18, 25 de enero]; } \\
\text { Nacimiento de la } \\
\text { biopolitica } \text { (1978- } \\
\text { 1979) [como } \\
\text { proyecto] }\end{array}$ & $\begin{array}{l}\text { Agamben en } \\
\text { Homo Sacer I } \\
\text { (1995) y Medios } \\
\text { sin fin (1996); } \\
\text { Negri y Hardt } \\
\text { en Imperio } \\
\text { (2000), y un } \\
\text { artículo de } \\
\text { Deleuze: } \\
\text { "Postescritum a } \\
\text { las sociedades } \\
\text { de control" } \\
\text { (1990) }\end{array}$ & $\begin{array}{l}\text { Politicas del } \\
\text { acontecimiento de } \\
\text { Maurizio Lazzarato } \\
\text { (2006); Bios: bipolítica y } \\
\text { filosofia (2006) y } \\
\text { Comunidad, inmunidad y } \\
\text { biopolítica (2009) de } \\
\text { Roberto Esposito; } \\
\text { Biopolíticas y formas de } \\
\text { vida editado por Rubén } \\
\text { Sánchez (2007); La } \\
\text { fábrica de porcelana de } \\
\text { Negri (2008); e Historia de } \\
\text { la gubernamentalidad de } \\
\text { Castro-Gómez (2010) }\end{array}$ & $\begin{array}{l}\text { No es posible establecer una cantidad } \\
\text { determinada del archivo, ni siquiera } \\
\text { representativa, de los trabajos sobre } \\
\text { este concepto; sin embargo, es } \\
\text { posible sostener que todos ellos } \\
\text { tienen una generalidad: usan los } \\
\text { conceptos tanato, anatomo, } \\
\text { biopolítica y biopoder para describir } \\
\text { casos concretos, que van desde la } \\
\text { didáctica y la pedagogía, la medicina, } \\
\text { la política, etc,. Al respecto se puede } \\
\text { observar el volumen de trabajos } \\
\text { presentados en el IV Coloquio } \\
\text { latinoamericano de biopolítica y el II } \\
\text { Coloquio internacional de biopolítica } \\
\text { y educación, así como la página } \\
\text { www.biopolitica.org }\end{array}$ \\
\hline
\end{tabular}




\title{
LA TRADICIÓN DE LA GUBERNAMENTALIDAD
}

\begin{abstract}
El manejo de los «procesos biosociológicos de las masas humanas», a diferencia de las disciplinas, puestas en práctica en el marco de instituciones limitadas (escuela, hospital, cuartel, taller, etcétera), implica el aparato estatal. Los «órganos complejos de coordinación y centralización» necesarios para este fin se encuentran en el nivel del Estado. La biopolítica, en consecuencia, sólo puede concebirse como una «birregulación por el Estado» (Senellart, 2006, p. 438).
\end{abstract}

\section{Primer momento: Las clases de Foucault}

Desde la cuarta clase de Seguridad, territorio, población (STP), 1 de febrero de 1978, Foucault introduce el concepto de gubernamentalidad; en las clases precedentes había trabajado sobre una perspectiva general del funcionamiento de los mecanismos de poder suscritos en el biopoder; de hecho, había continuado en este texto el proyecto iniciado en los setenta, cuando había comenzado a trabajar sobre las tecnologías políticas. De este modo, en STP su preocupación es sobre el tránsito de la tanatopolítica, esto es, del sobrepoder del soberano cuyo objetivo primordial era extraer el máximo de bienes y riquezas a través del control del territorio, hacia la biopolítica, cuyo objetivo primordial es extraer el máximo de ganancias a través de la menor intervención en la regulación de la población.

Así pues, la pregunta que guiaba el curso era: ¿podemos decir que en nuestras sociedades la economía general del poder está pasando a ser del orden a la seguridad?, y esta pregunta guía se siguió a través de la grilla de inteligibilidad de las técnicas de seguridad (técnicas biopolíticas) y su correlación con la población; sin embargo, esta noción se convirtió en el punto de quiebre de la indagación, porque condujo, a su vez, a la pesquisa por el «gobierno», concepto que designa, según Senellart (2006), las técnicas específicas de manejo de las poblaciones. De este modo, Foucault aborda en la cuarta clase de STP el estudio del «gobierno» e introduce el concepto de gubernamentalidad. Este concepto, desde el punto de vista originario en la obra de Foucault, debe entenderse en, al menos, tres sentidos:

Con esta palabra, «gubernamentalidad», aludo a tres cosas. Entiendo el conjunto constituido por las instituciones, los procedimientos, análisis y reflexiones, los cálculos y las tácticas 
que permiten ejercer esa forma bien específica, aunque muy compleja, de poder que tiene por blanco principal la población[;] por forma mayor de saber[,] la economía política[,] y por instrumento técnico esencial[,] los dispositivos de seguridad. Segundo, por «gubernamentalidad» entiendo la tendencia, la línea de fuerza que, en todo Occidente, no dejó de conducir, y desde hace mucho, hacia la preeminencia del tipo de poder que podemos llamar «gobierno» sobre todos los demás: soberanía, disciplina, y que indujo, por un lado, el desarrollo de toda una serie de aparatos específicos de gobierno, [y por el otro] el desarrollo de toda una serie de saberes. Por último, creo que habría que entender la «gubernamentalidad» como el proceso o, mejor, el resultado del proceso en virtud del cual el Estado de justicia de la Edad Media, convertido en Estado administrativo durante los siglos XV y XVI, se «gubernamentalizó» poco a poco (Foucault, 2006, p. 136).

El punto de quiebre que establece este concepto cambia el proyecto del texto; incluso, el mismo Foucault alega: «En el fondo, si hubiese querido dar al curso propuesto este año un título más exacto, con seguridad no habría elegido 'Seguridad, territorio, población'. Lo que querría hacer ahora, si tuviera verdadero interés en hacerlo, es algo que llamaría una historia de la 'gubernamentalidad'» ${ }^{6}(2006$, pp. 135-136).

Foucault desarrolla en STP esa genealogía de la gubernamentalidad: «La pastoral, la nueva técnica diplomático-militar y, por último, la policía fueron, a mi entender, los tres grandes puntos de apoyo sobre cuya base pudo producirse ese fenómeno fundamental en la historia de Occidente que fue la gubernamentalización del Estado» (Foucault, 2006, p. 138).

En cuanto a Nacimiento de la biopolítica, es una continuación del proyecto de STP y no debe entenderse, como sugiere el título, como una genealogía de la biopolítica ${ }^{7}$. El plan anunciado era estudiar el liberalismo, para

\footnotetext{
${ }^{6}$ Es la primera vez que aparece el término en la obra de Foucault.

${ }^{7}$ «El estudio anunciado de los mecanismos por los cuales la especie humana ingresó, en el siglo XVIII, en una estrategia general del poder, presentado como el esbozo de «una historia de las tecnologías de seguridad», cede su lugar, desde la cuarta clase del curso de 1978, al proyecto de una historia de la «gubernamentalidad» desde los primeros siglos de la era cristiana. Del mismo modo, el análisis de las condiciones de formación de la biopolítica, en el segundo curso, se borra al punto en beneficio del examen de la gubernamentalidad liberal. [...] Pero como efecto de esta investigación se desplaza al 
luego pasar al problema de la política de la vida; mas no fue así, la introducción se convirtió en todo el problema. En esas clases, «Foucault explicita en primer lugar el lazo entre verdad y gubernamentalidad liberal, a través del análisis del mercado como ámbito de veridicción» (Senellart, 2007, pp. 368-369).

Hasta acá podría comprenderse inadecuadamente que no hay una relación entre la tradición biopolítica y la tradición gubernamental de Foucault, sin embargo, es él mismo quien aclara en qué consiste la relación:

Pero me parece que el análisis de la biopolítica solo puede hacerse cuando se ha comprendido el régimen general de esa razón gubernamental de la que les hablo, ese régimen general que podemos llamar cuestión de la verdad, primeramente de la verdad económica dentro de la razón gubernamental; y por ende, si se comprende con claridad de qué se trata en ese régimen que es el liberalismo, opuesto a la razón de Estado [...] una vez que se sepa qué es ese régimen gubernamental denominado liberalismo, se podrá, me parece, captar qué es la biopolítica (Foucault, 2007, p. 41).

mismo tiempo el centro de gravedad de los cursos de la cuestión del biopoder a la cuestión del gobierno, a tal extremo que la segunda, en definitiva, eclipsa casi por completo la primera» (Senellart, 2006, pp. 417-418).

El $\boldsymbol{N B}$ iba a ser un curso dedicado exclusivamente a la biopolítica, y en la primera clase Foucault hace evidente esa intención: «Creí que este año podía hacer un curso sobre la biopolítica. Trataré de mostrarles que todos los problemas que intento identificar actualmente tienen como núcleo central, por supuesto, ese algo que llamamos población. Por consiguiente, será a partir de allí que pueda formarse algo semejante a una biopolítica» (Foucault, 2007, pp. 40-41).

Incluso en la cuarta clase de ese curso no había alcanzado el tema de la biopolítica: «si la suerte me sonríe, llegaremos al problema de la biopolítica y el problema de la vida» (Foucault, 2007, p. 97). Y ya al comienzo de la clase séptima, Foucault asegura que tenía la intención de hablar sobre biopolítica, pero esa intención no se cumplió: «Les aseguro que, pese a todo, en un comienzo tuve en verdad la intención de hablarles de biopolítica, pero después, como las cosas son lo que son, resulta que terminé por hablarles extensamente -demasiado extensamente, tal vez- del neoliberalismo, y además del neoliberalismo en su forma alemana» (Foucault, 2007, pp. 217).

Finalmente, «la suerte no le sonrió». En el «Resumen del curso» de $\boldsymbol{N B}$, que Foucault escribió después de haber terminado sus clases: «El curso de este año se dedicó finalmente, en su totalidad, a lo que sólo debía ser su introducción. El tema seleccionado era, entonces, la biopolítica» (Foucault, 2007, p. 359). 
Así que no debe entenderse como dos formas de ver las relaciones de poder, sino como dos accesos metodológicos al mismo problema, y esto es claro si se comprende que parte de las críticas que se le habían hecho a Foucault era la forma tan precaria de ver las relaciones macro, en cuanto todo su proyecto (70-77) había consistido en revisar las formas como se construyen las relaciones de poder en espacios micro. Con esta nueva «grilla de inteligibilidad» que se establece con la gubernamentalidad, Foucault podrá pensar en los elementos macro a través del Estado y la Población.

\begin{abstract}
¿Por qué estudiar ese dominio en definitiva inconsistente y brumoso, recubierto por una noción tan problemática y artificial como la de «gubernamentalidad»? Mi respuesta, inmediata y segura será la siguiente: para abordar el problema del Estado y la población. [...] La noción de Estado y población tienen su historia. El dominio al cual se refieren es más o menos conocido en términos generales, o aunque tenga una parte sumergida y oscura, tiene otra que es visible. Entonces como se trata de estudiar ese dominio a lo sumo (o para colmo) semioscuro del Estado y la población [...] (Foucault, 2006, p. 140).
\end{abstract}

La pretensión de estudio es, entonces, cómo funcionan las tecnologías políticas de la vida en un régimen de comprensión más amplio que involucra no solo las instituciones y las esferas micro, sino una proyección mayor, una esfera macro, las racionalidades gubernamentales.

\title{
El lado semioscuro del Estado: cuestiones de método
}

Algunas de las clases de STP están destinadas a especificar una racionalidad, la razón de Estado, mientras el Nacimiento de la biopolítica hablará sobre el cambio que se dio en esa razón de Estado y a lo que Foucault llamará razón gubernamental. Él establecerá que la razón gubernamental no es más que el perfeccionamiento de la razón de Estado.

Tanto en STP como en el Nacimiento de la biopolítica se desarrollará una postura del «gobierno de los hombres, en la medida -y sólo en la medidaen que se presenta como ejercicio de la soberanía política.» (Foucault, 2007, p. 17). Pero con esto él no pretende hacer un análisis de la práctica gubernamental real, su trabajo consiste en investigar el arte de gobernar, es decir, «la manera meditada de hacer el mejor gobierno y también, y al mismo 
tiempo, la reflexión sobre la mejor manera posible de gobernar» (Foucault, 2007, p. 17), y agrega:

De cómo dentro y fuera del gobierno y, en todo caso en la mayor contigüidad posible con la práctica gubernamental, se intentó conceptualizar esa práctica consistente en gobernar. Querría determinar de qué modo se estableció el dominio de la práctica del gobierno, sus diferentes objetos, sus reglas generales, sus objetivos de conjunto para gobernar de la mejor manera posible. En suma, es el estudio de la racionalización de la práctica gubernamental en el ejercicio de la soberanía política (Foucault, 2007, p. 17).

Esa decisión de hablar sobre las prácticas gubernamentales implica una elección de método. Se trata, entonces, de estudiar las prácticas tal y como se presentan y estudiar su racionalización, y a partir de eso determinar cómo se constituyen en los hechos el Estado, el soberano, los súbditos, es decir, aquello que Foucault llamará los universales con los que trabajan tanto el análisis de la filosofía política, como el de la sociología y la historia.

La razón de Estado es la racionalidad que permite ajustar la manera de gobernar el Estado; este tomado como dato que ya de hecho se presenta, pues «sólo se gobierna un Estado que se da como ya presente», pero también como algo por construir, algo que aún no está acabado. Lo que haría allí el arte de gobernar es racionalizar las maneras de obrar de ese Estado y transformar la manera como funciona en su deber ser, esto es, en una forma ideal en que debería operar el Estado. A esto Foucault lo llamará el arte de gobernar; este tendrá formas específicas para que el Estado llegue a ser lo que debe ser, una de ellas es la razón de Estado, y otra, la razón gubernamental. A la primera se dedicó STP, y a la segunda, Nacimiento de la biopolítica.

De esta manera, Foucault explica, a través de las prácticas, cómo se desarrolla, cómo conquista, cómo se encoge o extiende el Estado, qué tipo de racionalidad hace que este se transforme, cambie; con lo cual no puede ser visto como un universal que permitiría hacer la historiografía de las prácticas estatales, sino a la inversa, serían las prácticas las que permiten determinar la historiografía del Estado. No es un monstruo frío que aparece como dato histórico a-priori, sino que, justamente, es una práctica que se construye, se forma y mantiene a través de la experiencia, a través de las acciones, de los 
mecanismos, de las tácticas, de las técnicas, de las estrategias, que en última instancia se constituyen en un cuerpo organizado, en una racionalidad.

El derecho, la práctica judicial en la Edad Media, junto con el ejército, se había convertido en el multiplicador del poder real. A partir del siglo XVII y, sobre todo, a comienzos del siglo XVIII, cuando se desarrolla ese nuevo arte de gobernar, el derecho se convertirá en esa limitante de la razón de Estado que cobra cuerpo en un Estado de policía; este es el tránsito de la razón imperial a la razón de Estado.

El derecho, entonces, cambiará sus funciones. En primer lugar, ya no será multiplicador del poder soberano, sino sustractor de ese poder. En cierto sentido, los juristas dirán que hay una serie de leyes que preceden, que son anteriores al Estado, pues son constitutivas de este, y, por lo tanto, debe someterse a ellas. Estas leyes constitutivas también limitarán el poder del soberano (que en la razón imperial estaba concentrado exclusivamente en el rey).

Finalmente, la economía política será a la razón de Estado lo que el derecho es a la razón imperial, esto es, su limitante. La economía política, y especialmente el liberalismo, pondrá cada vez más límites al Estado. Otros mecanismos mucho más diversificados serán los que actúen bajo el principio de gobernar lo menos posible para obtener el máximo beneficio; contrario a la razón imperial y a la razón estatal, en las que se debía gobernar lo máximo posible para obtener, en la primera, el mayor número de bienes y riquezas, y en la segunda, el máximo control y la máxima productiva.

Así, Foucault podrá pasar de la razón imperial a la razón estatal, y de esta, a la razón gubernamental. Este proyecto sufre unas modificaciones en la clase de 1980, «Del gobierno de los vivos», pues ya no se trata de la forma oscura como gobierna el Estado, sino de la forma como el sujeto puede gobernarse a sí mismo:

Le cours de cette année a pris appui sur les analyses faites les années précédentes à propos de la notion de «gouvernement»: cette notion étant entendue au sens large de techniques et procédures destinées à diriger la conduite des hommes. Gouvernement des enfants, gouvernement des âmes ou des 
consciences, gouvernement d'une maison, d'un État ou de soimême. À l'intérieur de ce cadre très général, on a étudié le problème de l'examen de conscience et de l'aveu ${ }^{8}$ (Foucault, 2001).

Quiero agregar un último punto sobre esta posible digresión entre la biopolítica y la gubernamentalidad en la obra de Foucault. Algunos podrían interpretar en Foucault una hipótesis represiva antes de los cursos sobre la gubernamentalidad; esta lectura sería inconveniente porque no permitiría comprender el funcionamiento efectivo de las tecnologías políticas. Habría que preguntarse, por ejemplo, si la anatomopolítica no es un gobierno sobre acciones, en caso tal de que se leyera en la clave interpretativa de un análisis bélico que implica solo dominación/represión. A mi parecer, una línea interpretativa de la obra de Foucault podría mostrar que esta tecnología anatomopolítica es, al igual que la biopolítica, un gobierno sobre acciones, solo que se ejerce con mecanismos distintos (directamente/a distancia, sobre el cuerpo/sobre la población, predominantemente opresiva/predominantemente «libertaria», con regulación jurídica/con regulación estadística, normativización/normalización).

Creo que la lectura bélica frente a la lectura de juego de acciones sobre acciones, dominio vs. dirección, violencia vs. producción, represión vs. guía, no permite ver el funcionamiento efectivo de las relaciones de poder. Lo más positivo sería decir que en las tres racionalidades (racionalidad imperial, racionalidad estatal, racionalidad gubernamental) y en las tres tecnologías que las acompañan (tanatopolítica, anatomopolítica, biopolítica) gobernar la conducta significa cosas distintas, en tanto sus racionalidades (objetivos, medios técnicos para alcanzar esos objetivos, las reflexiones teóricas para alcanzar esos objetivos, y las estrategias que permiten la articulación entre medios y fines) son distintas.

No se trata, en consecuencia, de comprender las gubernamentalidades a partir de un análisis exterior de lo que sería el poder para Foucault en una etapa $\mathrm{u}$ otra de su pensamiento, sino de comprender cómo funcionan

\footnotetext{
${ }^{8}$ El curso de este año se ha basado en el análisis, realizado en los años precedentes, sobre el concepto de "gobierno»: este concepto se entiende en el sentido amplio de técnicas y procedimientos para regir la conducta de los hombres. Gobierno de los niños, el gobierno de las almas y conciencias, el gobierno de una casa, de un Estado o de uno mismo. Dentro de este marco general, se había estudiado el problema del autoexamen y la confesión (Traducción libre).
} 
efectivamente esas racionalidades. Esto permite superar la dicotomía dominadodominador y entender las relaciones de poder en su efectividad, en una dinámica de fuerzas que se relacionan, que pueden ser variables, modificables y reversibles. No se trata de que en un momento de la historia las racionalidades ejercían el poder y no había espacios de libertad, mientras en otra hay espacios de libertad y no se ejerce el poder.

En resumen, se puede interpretar una continuidad entre la biopolítica y la gubernamentalidad. La diferencia de matiz puede ser reinterpretada, en la obra de Foucault, a la luz de un acceso metodológico distinto a un mismo problema.

\section{Segundo momento de la gubernamentalidad: los discípulos de Foucault}

Un segundo momento de la gubernamentalidad puede ser distinguido con los intentos de superación de los vacíos que la obra de Foucault dejó sobre los temas que abordamos en la sección anterior, especialmente sobre el liberalismo, del cual Foucault dejó dos siglos sin estudiar; François Ewald(1991), Daniel Defert (1991), Jacques Donzelot $(1979,2007)$ y Giovanna Procacci (1991, 1998) - todos ellos discípulos de Foucault- «asumieron la tarea de llenar este espacio vacío de casi dos siglos y empezaron a reflexionar sobre las tecnologías liberales de gobierno en el siglo XIX» (Castro-Gómez, 2010, p. 229).

Esta serie de discusiones en torno a los vacíos sobre el liberalismo en la obra de Foucault han desarrollado un ámbito especial en el tipo de interpretaciones políticas que se le pueden asociar. Por un lado, una porción considerable de lectores creen que Foucault sirve como base teórica de apoyo a la izquierda intelectual y su lucha en contra del neoliberalismo; no obstante, este tipo de lecturas son poco críticas, y algunas veces olvidan rápidamente el análisis profundo de los textos por la necesidad de hacer uso de la teoría para describir casos concretos o para realizar luchas ideológicas.

Otras lecturas son más mesuradas y ven en la obra de Foucault una descripción o un diagnóstico, más que una crítica o una solución a un problema. En realidad, los textos de Foucault, especialmente los de la segunda mitad de los setenta, exigen una lectura detenida, rigurosa, meticulosa en el trasfondo y en las palabras, para poder argumentar una posición sobre el apoyo o no al neoliberalismo. No hay allí, al menos de manera superficial o en un primer plano, una posición definida. 
Otras lecturas son más arriesgadas e invitan a pensar críticamente la lectura que Foucault hace sobre el neoliberalismo. Así lo hace Daniel Zamora en una entrevista publicada en la revista Jacobin, «Can We Criticize Foucault?» (2014), que fue traducida y publicada al español por la revista Ando en Pando (2015); Zamora plantea que puede darse una interpretación de un Foucault cercano al neoliberalismo:

Foucault estuvo altamente atraído al liberalismo económico: él vio allí la posibilidad de una forma de gubernamentabilidad que era mucho menos normativa y autoritaria que la de la izquierda socialista y comunista, a la que él veía como totalmente obsoleta. Él vio especialmente en el neoliberalismo una forma de política «mucho menos burocrática»y «mucho menos disciplinaria» que aquella ofrecida por el Estado de bienestar de la posguerra. Él parecía imaginar un neoliberalismo que no proyectara sus modelos antropológicos en el individuo, que pudiera ofrecer a los individuos una mayor autonomía de cara al Estado (Zamora, 2015).

Independientemente de si Foucault apoyaba o no el neoliberalismo, si sus posturas son cercanas a la izquierda radical o no, lo interesante acá es la tradición abierta a nuevos desafíos hermenéuticos, a nuevas modalidades de comprensión y espacios de estudio.

\section{Tercer momento de la gubernamentalidad: los «Guvernamentality Studies»}

Un tercer momento de la gubernamentalidad puede ser caracterizado por los «Guvernamentality Studies», que se establecen, según Castro-Gómez, con la creación en Londres de la red «Historia del presente», en 1989. Los nombres más representativos de los estudios de la gubernamentalidad, según Castro-Gómez, son: Peter Miller (2008), Nikolas Rose (1999, 2004, 2007, 2012), Colin Gordon (1991), Graham Burchell (1996), Mitchel Dean (1994, 2008) y Majia Holmer Nadesan (2008) -solo uno de ellos perteneció a uno de los grupos de investigación que dirigió Foucault-. Esta red internacional de investigadores tiene sus nichos en Inglaterra, Canadá, Estados Unidos y Australia, y marginalmente se presentan algunos escritores latinoamericanos, algunos de ellos asociados a la Red Biopolítica. 
Dentro de este mismo momento se deben ubicar los trabajos que el propio Castro-Gómez ha efectuado (2009) y que ha liderado $(2004,2008)$, o ha difundido (Cortés Salcedo y Marín Díaz, 2011); así como también algunos de los trabajos de Oscar Saldarriaga (1997) y en particular las investigaciones realizadas en el instituto PENSAR de la Universidad Javeriana.

Sobre los dos últimos momentos de esta tradición, el capítulo anexo a Historia de la gubernamentalidad, «Historia de la gubernamentalidad después de Foucault», muestra un panorama detallado y ejemplifica la forma como los estudios de la gubernamentalidad están trabajando. El texto de Castro-Gómez hace un análisis detallado de la obra de Foucault y va más allá al correlacionar y ampliar el panorama del liberalismo y, especialmente, del neoliberalismo, al que Foucault no alcanzó a desarrollar en plenitud, pero del cual Historia de la gubernamentalidad hace un aporte significativo.

Cuadro 2. La tradición gubernamental

\begin{tabular}{|c|c|c|c|}
\hline \multicolumn{4}{|c|}{ LA TRADICIÓN GUBERNAMENTAL } \\
\hline Primer momento: Foucault, seten & & $\begin{array}{l}\text { Segundo momento: } \\
\text { noventas }\end{array}$ & $\begin{array}{l}\text { Tercer momento: primera década del } \\
2000\end{array}$ \\
\hline Seguridad, territorio, población & Nacimiento de la biopolítica & Vacios del liberalismo & Guvernmentality Studies \\
\hline $\begin{array}{l}\text { Desde la cuarta clase: } \\
\text { Introducción del concepto de } \\
\text { gubernamentalidad. Pastoral } \\
\text { cristiana y con ello la } \\
\text { gubernamentalización del } \\
\text { Estado, clases quinta a octava } \\
\text { [8, 15, } 22 \text { de febrero y } 1 \text { de } \\
\text { marzo]. El paso del pastorado al } \\
\text { gobierno político de los } \\
\text { hombres, clase novena [ } 8 \text { de } \\
\text { marzo]. El arte de gobernar } \\
\text { según la razón de Estado, final } \\
\text { de la novena hasta la undécima } \\
\text { clase [del } 8 \text { al } 22 \text { de marzo]. La } \\
\text { policía, duodécima y décimo } \\
\text { tercera clases [ } 29 \text { de marzo y } 5 \\
\text { de abril]. La última clase se } \\
\text { dedica al problema de la } \\
\text { población [5 de abril] }\end{array}$ & $\begin{array}{l}\text { Primera clase [10 de enero] cuestiones de } \\
\text { método y resumen del curso STP; segunda } \\
\text { clase y tercera [17 y } 24 \text { de enero] estudio de } \\
\text { los rasgos específicos del arte liberal de } \\
\text { gobernar. En la tercera clase se examina la } \\
\text { cuestión de Europa y sus relaciones con el } \\
\text { resto del mundo de acuerdo con la nueva } \\
\text { razón gubernamental que implica el } \\
\text { liberalismo. Desde la cuarta clase hasta la } \\
\text { décima [ } 31 \text { de enero; } 7,14,21 \text { de febrero y } 7 \\
\text { de marzo] el curso es dedicado al estudio de } \\
\text { las grandes escuelas neoliberales: el } \\
\text { ordoliberalismo alemán y el } \\
\text { anarcoliberalismo norteamericano como } \\
\text { formas críticas de la irracionalidad del } \\
\text { exceso de gobierno. Las dos últimas clases } \\
\text { se ocupa de la genealogía del homo } \\
\text { economicus, así como de formular las } \\
\text { conclusiones generales de la crisis del } \\
\text { liberalismo o su paradoja. }\end{array}$ & $\begin{array}{l}\text { François Ewald (1991), } \\
\text { Daniel Defert (1991), } \\
\text { Jacques Donzelot (1979), } \\
\text { (2007) ; y Giovanna } \\
\text { Procacci (1991), (1998) }\end{array}$ & $\begin{array}{l}\text { Peter Miller (2008) ; Nikolas Rose } \\
\text { (1999), (2004), (2007), (2012); Colin } \\
\text { Gordon (1991), Graham Burchell } \\
\text { (1996), Mitchel Dean (1994), } \\
\text { (2008); Majia Holmer Nadesan } \\
\text { (2008); Castro-Gómez (2009), } \\
\text { (2004), (2008); Oscar Saldarriaga } \\
\text { (1997) y en particular las } \\
\text { investigaciones realizadas en el } \\
\text { instituto PENSAR de la Universidad } \\
\text { Javeriana. }\end{array}$ \\
\hline
\end{tabular}

En resumen, la tradición biopolítica está atravesada por un momento de formación en la obra de Foucault, cercano a la década de los setenta; un momento de primera expansión, a finales de la década de los noventa; un momento de conformación discursiva, en la primera década del 2000, y un momento de implosión en las ciencias sociales, políticas y humanas, en la segunda década del 2000. Así mismo, la tradición gubernamental presenta tres momentos: 
el primero, en la etapa inicial de conformación en la obra de Foucault, en la segunda mitad de la década de los setenta; el segundo, identificado por el intento de superación de los vacíos sobre el liberalismo realizado por algunos discípulos de Foucault, en la década de los noventa, y, finalmente, los Guvernmentality Studies, desde el 2000. Ambas tradiciones no pueden ser comprendidas por separado, pues obedecen a un mismo proyecto intelectual que tiene divergencias metodológicas.

\section{REFERENCIAS}

Agamben, G. (1998). Homo Sacer I. El poder soberano y la nuda vida. Valencia: Pretextos.

Agamben, G. (2001). Medios sin fin: Notas sobre política. Valencia: Pre-textos.

Burchell, \& Graham. (1996). Liberal Goverment and the Techniques of the self. En A. Barry, T. Osborne, \& N. Rose (Edits.), Foucault and Political Reason: Liberalism, Neoliberalism and Racionalities of Goverment.

Castro-Gómez, S. ( 2009). Tejidos oníricos. Movilidad, capitalismo y biopolitica en Bogotá (1910-1930). Bogotá: Pontificia Universidad Javeriana.

Castro-Gómez, S. (2010). Historia de la gubernamentalidad. Razón de Estado, liberalismo y neoliberalismo en Michel Foucault. Bogotá: Siglo del Hombre; Pontificia Universidad Javeriana; Universidad Santo Tomás de Aquino.

Castro-Gómez, S. (Ed.). (2004). Pensar el Siglo XX. Pittsburgh: Universidad de Pittsburgh.

Castro-Gómez, S. y Restrepo, E. (Eds.). (2008). Genealogías de la colombianidad. Formaciones discursivas y tecnologías de gobierno en los siglos XIX y XX. Bogotá: Pontificia Universidad Javeriana.

Colin, G. (1991). Governmental Rationality: An Introduccion. En B. Graham, G. Colin, \& P. Miller (Eds.), The Foucault Effect: Studies in Governmentality. Chicago: The University of Chicago Press.

Cortés Salcedo, R. A. y Marín Díaz, D. L. (2011). Gubernamentalidad y educación. Discusiones contempóraneas. Bogotá: IDEP. 
Dean, M. (1994). Critical and Effective Histories: Foucault's Methods and Historical Sociology. New York: Routledge.

Dean, M. (1999. ). Governmentality. Power and Rule in Modern Society. Los Angeles: Sage Publications.

Defert, D. (1991). Popular Life and Insurance Technology. En C. G. Burchel, \& P. M. (eds.), The Foucault Effect. Studies in Governmentality. Chicago: The University of Chicago Press.

Deleuze, G. (1996). Postscritum a las sociedades de control. En G. Deleuze, Conversaciones. Valencia: Pretextos.

Donzelot, J. (1979). La policía de las familias. Valencia: Pre-Textos.

Donzelot, J., \& Cardoso, H. (2007). La invencioin de lo social: ensayo sobre la declinacioin de las pasiones poliiticas. Buenos Aíres: Nueva Visión.

Donzelot, J., \& Gordon, C. (Enero de 2008). Governing Liberal Societies. The Foucault effect in the English-speaking World. The Foucault Studies(5).

Eribon, D. (1992). Michel Foucault. Barcelona: Anagrama.

Eribon, D. (1995). Michel Foucault y sus contemporáneos. Buenos Aires: Ediciones Nueva Visión.

Esposito, R. (2006). Bios: biopolítica y filosofía. Buenos Aires: Amorrortu.

Esposito, R. (2009). Comunidad, inmunidad y biopolítica. (A. Ruiz García, Trad.) Barcelona: Herder.

Ewald, F. (1991). Insurance and Risk. En B. Graham, C. Gordon, \& P. Miller (Edits.), The Foucault Effect. Studies in Governmentality. Chicago: The University of Chicago Press.

Foucault, M. (1967). Historia de la locura en la época clásica. (J. J. Utrilla, Trad.) México: FCE.

Foucault, M. (27 de Abril de 1978). La philosophie analytique du pouvoir. DE, III (232).

Foucault, M. (1991). Microfísica del poder ( $3^{\mathrm{a}}$ ed.). Madrid: La Piqueta.

Foucault, M. (Junio de 1995). Los juegos del poder. Meridiano (31). 
Foucault, M. (1999). Los intelectuales y el poder. Entrevista con Deleuze. En M. Foucault, Estrategias de poder. Obras esenciales Vol II. Barcelona: Paidós.

Foucault, M. (2000). Los Anormales. Curso en el Collège de France (19741975). (H. Pons, Trad.) Buenos Aires: Fondo de Cultura Económica.

Foucault, M. (2001). Du gouvernement des vivants. En M. Foucault, Dits et écrits II, 19761988 (pp. 944-948). París: Éditions Gallimard.

Foucault, M. (2001). El nacimiento de la clínica: una arqueología de la mirada médica (20 ed.). México: Siglo XXI.

Foucault, M. (2002). Defender la sociedad. Curso en el Collège de France (1975-1976). (H. Pons, Trad.) Buenos Aires: Fondo de Cultura Económica.

Foucault, M. (2003). El poder psiquiátrico. Curso en el Collège de France (1973-1974). (H. Pons, Trad.). Buenos Aires: Fondo de Cultura Económica.

Foucault, M. (2003). El yo minimalista y otras conversaciones. (T. p. Staps, Trad.). Buenos Aires: La Marca.

Foucault, M. (2006). Seguridad, territorio, población. Curso en el Collège de France (1977-1978). (H. Pons, Trad.). Buenos Aires: FCE.

Foucault, M. (2006). Vigilar y Castigar. (A. G. Camino, Trad.). Barcelona: Círculo de Lectores.

Foucault, M. (2007). Historia de la sexualidad 1: La voluntad de saber (31 ${ }^{\mathrm{a}}$ ed.). (U. Guiñazú, Trad.) México: Siglo XXI.

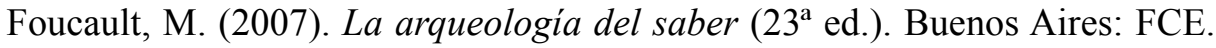

Foucault, M. (2007). Nacimiento de la biopolítica. Curso en el Collège de France (1978-1979). (H. Pons, Trad.). Buenos Aires: FCE.

Lazzarato, M. (2006). Políticas del acontecimiento. Buenos Aires: Tinta Limón.

Lazzarato, M. (s.f.). Del biopoder a la biopolítica. Recuperado el 02 de Septiembre de 2011, de http//multitudes.samizdat.net/ article.php3?id_article $=298$

Miller, P., \& Rose, N. (2008). Governing the Present. Administering Economic, Social and Personal Life. London: Polity Press. 
Morey, M. (1983). Lectura de Foucault. Madrid: Taurus.

Nadesan, M. H. (2008). Governmentality, Biopower, and Everyday Life. New York: Routledge.

Negri, A. (2008). La fábrica de porcelana. (S. Lauro, Trad.). Barcelona: Paidós.

Negri, A. y Hardt, M. (2005). Imperio. Barcelona: Paidós.

Negri, A. y Hardt, M. (2005). La producción biopolítica. En A. Negri, Imperio. Barcelona: Paidós.

Negri, A. y Hardt, M. (2011). Commonwealth: El proyecto de una revolución del común. Massachusetts: Harvard University Press.

Procacci, \& Giovanna. (1998). Governare la povertà: la società liberale e la nascita della questione sociale. Bologna: Società Editrice Il Mulino.

Procacci, G. (1991). Social Economy and the Government of poverty. En G. Burschell, C. Gordon, \& P. Miller (Edits.), The Foucault Effect: Studies in Governmentality. Chicago: The University of Chicago Press.

Rose, N. (1999). Governing the Soul. The Shaping of the Private Self. London: Free Association Books .

Rose, N. (2004). Powers of Freedom. Reframing Political Thought (Segunda ed.). Cambridge: Cambridge University Press.

Rose, N. (2007). The Politics of Life Itself. Biomedicine, Power and Subjectivity in the Twenty-First Century. Nueva Jersey: Princeton University Press.

Rose, N. (Junio de 2012). Gubernamentalidad. Astrolabio Nueva Época (8).

Rose, N., Avellaneda, A. y Vega, G. (2012). Governmentality Studies, liberalismo y control. Una entrevista con Nikolas Rose. Nuevo Itinerario: Revista digital de Filosofía, 7(VII).

Sáenz Obregón, J., Saldarriaga Vélez, Ó. y Ospina López, A. (1997). Mirar la infancia: pedagogía, moral y modernidad en Colombia, 1903-1946. Medellín: Universidad de Antioquia.

Sánchez, R. (2007). Alcances y límites de los conceptos biopolítica y biopoder en Michel Foucault. En R. Sánchez (Ed.), Biopolitica y formas de vida (pp. 17-43). Bogotá: Pontificia Universidad Javeriana. 
Sánchez, R. (Ed.). (2007). Biopolítica y formas de vida. Bogotá: Pontificia Universidad Javeriana.

Senellart, M. (2006). Situación de los cursos. En M. Foucault, Seguridad, Territorio, Población (pp. 417-453). Buenos Aires: FCE.

Senellart, M. (2007). Situación del curso. En M. Foucault, Nacimiento de la biopolitica (pp. 367-371). Buenos Aires: FCE.

Smart, B. (1998). Introductory Essay: The Government of Conduct -Foucault on Rationality, Power and Subjectivity. En B. Smart (Ed.), Michel Foucault, Critical Assessments (Vol. 4). London and New York: Routledge.

Zamora, D. (Diciembre de 2014). Jacobin. Obtenido de Can We Criticize Foucault?: https://www.jacobinmag.com/2014/12/foucault-interview/

Zamora, D. (Enero de 2015). Revista Ando en Pando. Obtenido de ¿Podemos criticar a Foucault? Foucault como neoliberal: https:// andoenpando.wordpress.com/2015/01/07/podemos-criticar-a-foucaultfoucault-como-neoliberal/ 
